\title{
Correction to: The therapeutic targeting of the FGFR1/Src/NF-KB signaling axis inhibits pancreatic ductal adenocarcinoma stemness and oncogenicity
}

\author{
Shiue-Wei Lai ${ }^{1,2,3}$. Oluwaseun Adebayo Bamodu ${ }^{4,5} \cdot$ Wen-Chiuan Tsai ${ }^{6,7} \cdot$ Yi-Ming Chang ${ }^{6,7} \cdot$ Wei-Hwa Lee $^{5,8}$. \\ Chi-Tai Yeh $^{9,10,11}$ (D) . Tsu-Yi Chao ${ }^{12,13,14}$
}

Published online: 4 January 2019

○) Springer Nature B.V. 2019

\section{Correction to: \\ Clinical \& Experimental Metastasis (2018) 35:663-677 \\ https://doi.org/10.1007/s10585-018-9919-5}

In the original publication, the affiliations of authors were published incorrectly. The corrected affiliations are given in this Correction.

The original article can be found online at https://doi.org/10.1007/ s10585-018-9919-5.

Chi-Tai Yeh

ctyeh@s.tmu.edu.tw

Tsu-Yi Chao

10575@s.tmu.edu.tw

1 Graduate Institute of Clinical Medicine, College of Medicine, Taipei Medical University, Taipei, Taiwan

2 Division of Hematology-Oncology, Department of Internal Medicine, Tri-Service General Hospital, National Defense Medical Center, Taipei, Taiwan

3 Department of Internal Medicine, Tri-Service General Hospital Penghu Branch, Penghu, Taiwan

4 Department of Hematology and Oncology, Cancer Center, Taipei Medical University-Shuang Ho Hospital, New Taipei City 23561, Taiwan

5 Department of Medical Research and Education, Taipei Medical University-Shuang Ho Hospital, New Taipei City 23561, Taiwan

6 Department of Pathology, Tri-Service General Hospital, National Defense Medical Center, Taipei, Taiwan

7 Graduate Institute of Medical Sciences, National Defense Medical Center, Taipei, Taiwan
8 Department of Pathology, Taipei Medical University-Shuang Ho Hospital, New Taipei City, Taiwan

9 Graduate Institute of Clinical Medicine, Taipei Medical University, Taipei, Taiwan

10 Department of Hematology and Oncology, Cancer Center, Taipei Medical University-Shuang Ho Hospital, New Taipei City 23561, Taiwan

11 Department of Medical Research and Education, Taipei Medical University-Shuang Ho Hospital, New Taipei City 23561, Taiwan

12 Graduate Institute of Clinical Medicine, Taipei Medical University, Taipei, Taiwan

13 Department of Hematology and Oncology, Cancer Center, Taipei Medical University-Shuang Ho Hospital, New Taipei City 23561, Taiwan

14 Department of Medical Research and Education, Taipei Medical University-Shuang Ho Hospital, New Taipei City 23561, Taiwan 